\title{
Systolic Time Interval (STI) in Hypothyroid Patients Receiving High Dose L-Thyroxine
}

Nurul Aulia Zakaria ${ }^{1}$, Hafizah Pasi ${ }^{1}$, Mohammad Arif Shahar ${ }^{2}$

1 International Islamic University Malaysia

${ }^{2}$ AVISENA Specialist Hospital Shah Alam

Introduction: Systolic Time Interval (STI) is a simple,noninvasive and precise technique to assess left ventricular (LV) function. It measures aortic Pre-Ejection Period (PEP) over Left Ventricular Ejection Time (LVET) from echocardiogram. Thyrotoxicosis will enhance LV function and cause reduction of STI. This study was perform to measure the changes of STI after administration of high dose L-thyroxine and to determine the correlation between high dose L-thyroxine administration and STI. Materials and Method: A Total of 22 patients were screened. Those with cardiac diseases and high Framingham risk score were excluded. Nine patients were started on high dose L-thyroxine (7x their usual dose) once a week during the month of Ramadan. Thyroid hormones ( T3,T4,TSH) $\hat{A}$ and STI (PEP/LVET) were measured at baseline and within $24 \mathrm{hrs}$ after high dose L-thyroxine ingestion. Results: All patients have normal thyroid hormones level and normal cardiac function at baseline. The median dose $(\mathrm{mcg})$ of L-thyroxine was $600(437.5,700)$ while the median level of fT4 $(\mathrm{pmol} / \mathrm{L})$ was $17.43(12.38,20.8)$. Despite the significant increment of fT4 after Lthyroxine ingestion [baseline $13.21(8.19,14.63)$ vs high dose $17.43(12.38,22.55) \mathrm{p}$; 0.011 ] there was no significant change in STI [baseline $0.3(0.2,0.4)$ vs high dose $0.28(0.26,0.45) \mathrm{p} ; 0.513]$. There was no correlation found between the dose of Lthyroxine and STI ( $r=0.244, p ; 0.526)$. Conclusion: Administration of high dose Lthyroxine did not significantly alter STI despite significant increment of fT4 level unlike the naturally occurring thyrotoxicosis. Therefore 'exogenous' administration of high dose L-thyroxine is cardiac safe. 